\title{
On the convergence of a regularizing Levenberg-Marquardt scheme for nonlinear ill-posed problems
}

\author{
Marlis Hochbruck • Michael Hönig
}

Received: July 21, 2009/ Accepted: date

\begin{abstract}
In this note we study the convergence of the Levenberg-Marquardt regularization scheme for nonlinear ill-posed problems. We consider the case that the initial error satisfies a source condition. Our main result shows that if the regularization parameter does not grow too fast (not faster than a geometric sequence), then the scheme converges with optimal convergence rates. Our analysis is based on our recent work on the convergence of the exponential Euler regularization scheme [3].
\end{abstract}

Keywords Nonlinear ill-posed problems · Levenberg-Marquardt method · optimal convergence rates

Mathematics Subject Classification (2000) 65J15 · 65J20

\section{Introduction}

In this note we study the convergence rates of the Levenberg-Marquardt method for solving the nonlinear ill-posed problem

$$
F(x)=y .
$$

Here $F: \mathcal{D}(F) \subset X \rightarrow Y$ is a nonlinear differentiable operator between the Hilbert spaces $X$ and $Y$, whose Fréchet derivative $F^{\prime}(u)$ is locally uniformly bounded. We always assume that (1) has a solution $x_{*} \in \mathcal{D}(F)$ but we do not assume that this solution is unique. We are interested in the case that only perturbed data $y^{\delta} \approx y$ satisfying

$$
\left\|y^{\delta}-y\right\| \leq \delta
$$

is available. Throughout the paper, the norm in both Hilbert spaces $X$ and $Y$ is denoted by $\|\cdot\|$, the corresponding inner product by $\langle\cdot, \cdot\rangle$.

It has been shown by Hanke [1] that the Levenberg-Marquardt method

$$
u_{n+1}=u_{n}+h_{n}\left(I+h_{n} J_{n}\right)^{-1} F^{\prime}\left(u_{n}\right)^{*}\left(y^{\delta}-F\left(u_{n}\right)\right), \quad n=0,1,2, \ldots
$$

M. Hochbruck, M. Hönig

Mathematisches Institut, Heinrich-Heine Universität Düsseldorf, D-40225 Düsseldorf, Germany 
with

$$
J(u)=F^{\prime}(u)^{*} F^{\prime}(u), \quad J_{n}=J\left(u_{n}\right)
$$

converges to a solution of the unperturbed problem (1) in the limit $\delta \rightarrow 0$ if the regularization parameter is chosen appropriately and if the iteration is stopped as soon as the standard discrepancy principle

$$
\left\|\Delta F_{n_{*}}^{\delta}\right\| \leq \tau \delta<\left\|\Delta F_{n}^{\delta}\right\| \quad \text { for all } n<n_{*},
$$

is satisfied for some parameter $\tau>1$. Hanke [1] suggests to select $h_{n}$ such that the following discrepancy principle

$$
\left\|\Delta F_{n}^{\delta}-F^{\prime}\left(u_{n}\right)\left(u_{n+1}-u_{n}\right)\right\|=\mu\left\|\Delta F_{n}^{\delta}\right\|, \quad \mu<1,
$$

is satisfied. Here

$$
\Delta F_{n}^{\delta}=y^{\delta}-F\left(u_{n}\right)
$$

denotes the residual of the perturbed problem.

Rieder $[7,8]$ managed to prove nearly optimal convergence rates for yet different adaptively chosen step sizes. Only recently, Jin [4] proved optimal convergence rates for an a priori chosen geometric step size sequence.

The aim of this note is to show that if the initial error satisfies a source condition, then the method converges with optimal rate for quite general step size sequences including the geometric sequence studied in [4]. Our analysis is based on our recent work [3], where we proved an analogous result for the exponential Euler regularization.

\section{Preliminaries}

In order to verify optimal convergence rates, certain assumptions have to be imposed. Let $x_{+}$be the solution of minimal distance to $x_{0}$. The following assumptions ensure, that this solution is unique, see [6, Proposition 2.1]. Our main assumption is that the initial error satisfies a source condition.

Assumption 1 There exists $w \in X$ and constants $\gamma \in(0,1 / 2]$ and $\rho \geq 0$ such that

$$
e_{0}=x_{0}-x_{+}=J\left(x_{+}\right)^{\gamma} w, \quad\|w\| \leq \rho .
$$

Moreover, we have to assume relations between the Fréchet derivatives evaluated at two different points in $B_{r}\left(x_{+}\right)$.

Assumption 2 For all $x, \widetilde{x} \in B_{r}\left(x_{+}\right)$there exist linear bounded operators $R(x, \widetilde{x})$ : $Y \rightarrow Y$ and a constant $C_{R} \geq 0$ such that

1. $F^{\prime}(x)=R(x, \widetilde{x}) F^{\prime}(\widetilde{x})$

2. $\|R(x, \widetilde{x})-I\| \leq C_{R}\|x-\widetilde{x}\|$.

Both assumptions are standard assumptions arising in the literature, see, e.g., [4-7]. Note, that for $C_{R} r<1 / 2$ Assumption 2 implies the so-called tangential cone condition

$$
\left\|F(\widetilde{x})-F(x)-F^{\prime}(x)(\widetilde{x}-x)\right\| \leq \eta\|F(x)-F(\widetilde{x})\|, \quad x, \widetilde{x} \in B_{r}\left(x_{+}\right) .
$$


with $\eta=C_{r} r /\left(1-C_{r} r\right)<1$, see, e.g., [7]. Moreover it is possiple to slightly weaken Assumption 2 by fixing $\widetilde{x}=x_{+}$. This results in a slightly larger constant of $3 / 2 C_{R}$ in (15) below, cf. equation (3.4) in [2].

To simplify the presentation we further assume without loss of generality that the problem is appropriately scaled, i.e.,

$$
\left\|F^{\prime}(x)\right\| \leq 1, \quad x \in B_{r}\left(x_{+}\right)
$$

\section{Convergence rates}

The aim of this section is to show that the Levenberg-Marquardt regularization in fact converges with optimal rates. Our results are valid under weak restrictions on the step sizes, namely we assume that there exist constants $c_{0}, c_{h}$ such that

$$
h_{0} \leq c_{0}, \quad 0<h_{j} \leq c_{h} t_{j}, \quad j \geq 1,
$$

where

$$
t_{0}=0, \quad t_{j+1}=t_{j}+h_{j}, \quad j=0,1,2, \ldots
$$

Note that this step size restriction allows to choose $\left(h_{j}\right)_{j \geq 0}$ as a geometric sequence and thus our result generalizes the recent result [4].

Theorem 1 Let Assumptions 1 and 2 hold and assume that the step sizes $h_{j}$ satisfy (9) for all $j \leq n_{*}$ and that $t_{j} \rightarrow \infty$ for $j \rightarrow \infty$. Here, the stopping index $n_{*}$ is defined by (5), with $\tau$ satisfying

$$
\tau>\frac{2-\eta}{1-\eta}
$$

Then for $\rho$ sufficiently small, the iterates $u_{n}$ stay in $B_{r}\left(x_{+}\right)$for $n=0,1, \ldots, n_{*}$ and the iteration stops after $n_{*}<\infty$ steps. Moreover, there exists a constant $C=$ $C\left(\tau, \eta, C_{R}, c_{0}, c_{h}, \gamma, r\right)>0$ such that

$$
\left\|u_{n_{*}}-x_{+}\right\| \leq C \rho^{1 /(2 \gamma+1)} \delta^{2 \gamma /(2 \gamma+1)} .
$$

The proof of this theorem is postponed to the end of this note.

Remark. The assumption $t_{j} \rightarrow \infty$ for $j \rightarrow \infty$ is satisfied if the step sizes are bounded away from zero or if they do not decay faster than $1 / j$, for instance.

Our analysis uses the discrete variation-of-constants formula (Theorem 2), which is derived from the following suitably written error recursion. Throughout the paper we denote the operators by

$$
\begin{aligned}
A_{+} & =F^{\prime}\left(x_{+}\right), & A_{n} & =F^{\prime}\left(u_{n}\right), \\
J_{+} & =A_{+}^{*} A_{+}, & J_{n} & =A_{n}^{*} A_{n}, \\
K_{+} & =A_{+} A_{+}^{*}, & K_{n} & =A_{n} A_{n}^{*},
\end{aligned}
$$

and the corresponding operator functions by

$$
\begin{array}{ll}
\Phi_{n,+}=\left(I+h_{n} J_{+}\right)^{-1}, & \Phi_{n}=\left(I+h_{n} J_{n}\right)^{-1} \\
\widetilde{\Phi}_{n,+}=\left(I+h_{n} K_{+}\right)^{-1}, & \widetilde{\Phi}_{n}=\left(I+h_{n} K_{n}\right)^{-1} .
\end{array}
$$


Lemma 1 Let Assumption 2 hold. Then the error

$$
e_{n}=u_{n}-x_{+}
$$

of the Levenberg-Marquardt recursion (3) satisfies

$$
e_{n+1}=\Phi_{n,+} e_{n}+h_{n} A_{+}^{*} \widetilde{\Phi}_{n,+}\left(r_{n}+y^{\delta}-y\right)
$$

where, for $R_{n}=R\left(u_{n}, x_{+}\right)$and $\widetilde{R}_{n}=R\left(x_{+}, u_{n}\right)$,

$$
r_{n}=F\left(x_{+}\right)-F\left(u_{n}\right)+A_{+} e_{n}+\left(R_{n}^{*}-I+\left(\widetilde{R}_{n}-R_{n}^{*}\right) \widetilde{\Phi}_{n} h_{n} K_{n}\right) \Delta F_{n}^{\delta} .
$$

If in addition the the stopping index $n_{*}$ is defined by (5), then there is a constant $C_{1}=C_{1}\left(\tau, \eta, C_{R}, c_{0}, c_{h}, \gamma, r\right)$ such that for $n<n_{*}$ we have

$$
\left\|r_{n}\right\| \leq C_{1}\left\|e_{n}\right\|\left\|A_{+} e_{n}\right\| .
$$

Proof By (3), the following error recursion holds

$$
\begin{aligned}
e_{n+1} & =\Phi_{n,+} e_{n}+h_{n} A_{+}^{*} \widetilde{\Phi}_{n,+}\left(F\left(x_{+}\right)-F\left(u_{n}\right)+A_{+} e_{n}\right) \\
+ & h_{n} \Phi_{n,+} A_{+}^{*}\left[\left(R_{n}^{*}-I\right)+h_{n}\left[\left(\widetilde{R}_{n}-I\right)-\left(R_{n}^{*}-I\right)\right] \widetilde{\Phi}_{n} K_{n}\right] \Delta F_{n}^{\delta} \\
+ & h_{n} \Phi_{n,+} A_{+}^{*}\left(y^{\delta}-y\right) \\
= & \Phi_{n,+} e_{n}+h_{n} A_{+}^{*} \widetilde{\Phi}_{n,+}\left\{F\left(x_{+}\right)-F\left(u_{n}\right)+A_{+} e_{n}+y^{\delta}-y\right. \\
& \left.\quad+\left[\left(R_{n}^{*}-I\right)+h_{n}\left[\left(\widetilde{R}_{n}-I\right)-\left(R_{n}^{*}-I\right)\right] \widetilde{\Phi}_{n} K_{n}\right] \Delta F_{n}^{\delta}\right\} .
\end{aligned}
$$

This proves the error recursion.

It was shown in [3, Lemma 4.3], that if the stopping index $n_{*}$ is defined by (5), then we have

$$
\left\|\Delta F_{n}^{\delta}\right\| \leq \frac{\tau}{(\tau-1)(1-\eta)}\left\|A_{+} e_{n}\right\|, \quad n<n_{*} .
$$

Moreover, equation (3.4) in [2] (for a slightly weaker form of Assumption 1) or [9, Proposition 4] yield

$$
\left\|F\left(x_{+}\right)-F\left(u_{n}\right)+A_{+} e_{n}\right\| \leq \frac{1}{2} C_{R}\left\|e_{n}\right\|\left\|A_{+} e_{n}\right\| .
$$

Defining

$$
C_{1}=C_{R}\left(\frac{1}{2}+3 \frac{\tau}{(\tau-1)(1-\eta)}\right)
$$

gives the bound (13).

Next we prove that the error norms $\left\|e_{n}\right\|$ and $\left\|A_{+} e_{n}\right\|$ decay with a rate proportional to $\left(1+t_{n}\right)^{\gamma}$ and $\left(1+t_{n}\right)^{\gamma+1 / 2}$, respectively.

Theorem 2 Let the assumptions of Theorem 1 hold. Then for $\rho$ sufficiently small there is a constant $C_{*}=C_{*}\left(\tau, \eta, C_{R}, c_{0}, c_{h}, \gamma, r\right)$ such that for $n \leq n_{*}$

$$
\begin{aligned}
\left\|e_{n}\right\| & \leq C_{*} \frac{\rho}{\left(1+t_{n}\right)^{\gamma}}, \\
\left\|A_{+} e_{n}\right\| & \leq C_{*} \frac{\rho}{\left(1+t_{n}\right)^{\gamma+1 / 2}} .
\end{aligned}
$$


Proof For an arbitrary $n \in \mathbb{N}$ the error recursion (12) leads to the following discrete variation-of-constants formulas

$$
\begin{aligned}
e_{n} & =\prod_{j=0}^{n-1} \Phi_{j,+} e_{0}+\sum_{j=0}^{n-1} h_{j} \prod_{k=j+1}^{n-1} \Phi_{k,+} A_{+}^{*} \widetilde{\Phi}_{j,+}\left(r_{j}+y^{\delta}-y\right) \\
& =\prod_{j=0}^{n-1} \Phi_{j,+} e_{0}+\sum_{j=0}^{n-1} h_{j} A_{+}^{*} \prod_{k=j}^{n-1} \widetilde{\Phi}_{k,+}\left(r_{j}+y^{\delta}-y\right) .
\end{aligned}
$$

Moreover, we have

$$
A_{+} e_{n}=A_{+} \prod_{j=0}^{n-1} \Phi_{j,+} e_{0}+\sum_{j=0}^{n-1} h_{j} K_{+} \prod_{k=j}^{n-1} \widetilde{\Phi}_{k,+}\left(r_{j}+y^{\delta}-y\right) .
$$

By Lemma 2 below, the sum multiplying $y^{\delta}-y$ in (16) can be bounded by

$$
\left\|\sum_{j=0}^{n-1} h_{j} A_{+}^{*} \prod_{k=j}^{n-1} \widetilde{\Phi}_{k,+}\right\| \leq \sum_{j=0}^{n-1} h_{j}\left(t_{n}-t_{j}\right)^{-1 / 2} \leq \int_{0}^{t_{n}} \frac{1}{\sqrt{t_{n}-x}} \mathrm{~d} x=2 \sqrt{t_{n}}
$$

while the corresponding sum in (17) can be bounded by one by using the identity

$$
\sum_{j=0}^{n-1} h_{j} K_{+} \prod_{k=j}^{n-1} \widetilde{\Phi}_{k,+}=I-\prod_{j=0}^{n-1} \widetilde{\Phi}_{j,+} .
$$

Thus, by Assumption 1, (13) and Lemma 2 we have

$$
\left\|e_{n}\right\| \leq \frac{\rho}{\left(1+t_{n}\right)^{\gamma}}+2 \sqrt{t_{n}} \delta+C_{1} \sum_{j=0}^{n-1} h_{j} \frac{1}{\sqrt{1+t_{n}-t_{j}}}\left\|e_{j}\right\|\left\|A_{+} e_{j}\right\|
$$

and

$$
\left\|A_{+} e_{n}\right\| \leq \frac{\rho}{\left(1+t_{n}\right)^{\gamma+1 / 2}}+\delta+C_{1} \sum_{j=0}^{n-1} h_{j} \frac{1}{1+t_{n}-t_{j}}\left\|e_{j}\right\|\left\|A_{+} e_{j}\right\| .
$$

Following the proof of Theorem 4.8 in [3], we proceed by induction for $n=$ $0,1, \ldots, n_{*}$. By Assumption 1, the statement is true for $n=0$ if $C_{*} \geq 1$. Assuming that the bounds hold for all indices up to $n-1$, we obtain

$$
\left\|e_{n}\right\| \leq \frac{\rho}{\left(1+t_{n}\right)^{\gamma}}+2 \sqrt{t_{n}} \delta+C_{*}^{2} \rho^{2} C_{1} S_{n}\left(\frac{1}{2}, 2 \gamma+\frac{1}{2}\right)
$$

and

$$
\left\|A_{+} e_{n}\right\| \leq \frac{\rho}{\left(1+t_{n}\right)^{\gamma+1 / 2}}+\delta+C_{*}^{2} \rho^{2} C_{1} S_{n}\left(1,2 \gamma+\frac{1}{2}\right)
$$

where

$$
S_{n}(\alpha, \beta)=\sum_{j=0}^{n-1} \frac{h_{j}}{\left(1+t_{n}-t_{j}\right)^{\alpha}\left(1+t_{j}\right)^{\beta}} .
$$

It was shown in Lemma 4.11 in [3], that the discrete sums can be bounded by

$$
S_{n}\left(\alpha, 2 \gamma+\frac{1}{2}\right) \leq C_{2} \frac{1}{\left(1+t_{n}\right)^{\alpha+\gamma-1 / 2}}
$$


provided that the step sizes satisfy (9). This leads to

$$
\begin{aligned}
\left\|e_{n}\right\| & \leq \frac{\rho}{\left(1+t_{n}\right)^{\gamma}}\left(1+C_{*}^{2} \rho C_{1} C_{2}\right)+2 \sqrt{t_{n}} \delta, \\
\left\|A_{+} e_{n}\right\| & \leq \frac{\rho}{\left(1+t_{n}\right)^{\gamma+1 / 2}}\left(1+C_{*}^{2} \rho C_{1} C_{2}\right)+\delta .
\end{aligned}
$$

By induction hypothesis and by applying (5) and (14) we get

$$
\begin{aligned}
\delta & \leq \frac{1}{(\tau-1)(1-\eta)}\left\|A_{+} e_{n-1}\right\| \\
& \leq \frac{1}{(\tau-1)(1-\eta)}\left(\frac{\rho}{\left(1+t_{n-1}\right)^{\gamma+1 / 2}}\left(1+C_{*}^{2} \rho C_{1} C_{2}\right)+\delta\right) .
\end{aligned}
$$

Using (9), we have

$$
\frac{1}{1+t_{n-1}} \leq \frac{1+c_{h}}{1+t_{n}}, \quad n=1,2, \ldots
$$

so that

$$
\delta \leq C_{3} \frac{\rho}{\left(1+t_{n}\right)^{\gamma+1 / 2}}
$$

with

$$
C_{3}=\frac{1+c_{h}}{(\tau-1)(1-\eta)-1}\left(1+C_{*}^{2} \rho C_{1} C_{2}\right)
$$

holds. Inserting this relation into (21) shows

$$
\begin{aligned}
\left\|e_{n}\right\| & \leq \frac{\rho}{\left(1+t_{n}\right)^{\gamma}}\left(1+C_{*}^{2} \rho C_{1} C_{2}+2 C_{3}\right), \\
\left\|A_{+} e_{n}\right\| & \leq \frac{\rho}{\left(1+t_{n}\right)^{\gamma+1 / 2}}\left(1+C_{*}^{2} \rho C_{1} C_{2}+C_{3}\right) .
\end{aligned}
$$

This yields the desired result, as long as

$$
1+C_{*}^{2} \rho C_{1} C_{2}+2 C_{3} \leq C_{*},
$$

holds, which can be achieved for $\rho$ sufficiently small.

In the previous proof, we have used the following estimate.

Lemma 2 For $0 \leq \alpha \leq 1$ we have

$$
\left\|K_{+}^{\alpha} \prod_{k=j}^{n-1} \widetilde{\Phi}_{k,+}\right\| \leq \min \left\{\left(t_{n}-t_{j}\right)^{-\alpha},\left(1+t_{n}-t_{j}\right)^{-\alpha}\right\} .
$$

Proof The inequality

$$
\prod_{k=j}^{n-1}\left(1+h_{k} \lambda\right) \geq 1+\lambda \sum_{k=j}^{n-1} h_{k}=1+\lambda\left(t_{n}-t_{j}\right)
$$

shows that

$$
\lambda^{\alpha} \prod_{k=j}^{n-1}\left(1+h_{k} \lambda\right)^{-\alpha} \leq\left(\frac{\lambda}{1+\lambda\left(t_{n}-t_{j}\right)}\right)^{\alpha} .
$$

For $x \in[0,1]$ the function $x /\left(1+x\left(t_{n}-t_{j}\right)\right)$ attains its maximum at $x=1$. This proves the second bound.

The first part of the bound was also used in [6, p. 109] or [4, Lemma 2]. 
Remark. If the maximum possible step sizes $h_{j}=c_{h} t_{j}, j=1, \ldots, n_{*}-1$ are chosen, then (14), Theorem 2, and (5) show that there is a constant $c$ such that the stopping index satisfies $n_{*} \leq c|\log \delta|$.

It remains to prove our main theorem.

Proof (of Theorem 1) By Theorem 2, the iterates $u_{n}$ stay in $B_{C_{*} \rho}\left(x_{+}\right)$for all $n=$ $0,1, \ldots, n_{*}$. Moreover, using (14) and $t_{n} \rightarrow \infty$, the bound of $\left\|A_{+} e_{n}\right\|$ also shows that the stopping index $n_{*}$ is finite.

In order to prove the convergence rate, we write (16) for $n=n_{*}$ in the form

$$
e_{n_{*}}=J_{+}^{\gamma} v_{*}+\sum_{j=0}^{n-1} h_{j} A_{+}^{*} \prod_{k=j}^{n_{*}-1} \widetilde{\Phi}_{k,+}\left(y^{\delta}-y\right),
$$

where

$$
v_{*}=\prod_{k=0}^{n_{*}-1} \Phi_{k,+} w+\sum_{j=0}^{n_{*}-1} h_{j} \prod_{k=j}^{n_{*}-1} \Phi_{k,+} J_{+}^{-\gamma} A_{+}^{*} r_{j} .
$$

Note that $v_{*}$ is well defined since

$$
J_{+}^{-\gamma} A_{+}^{*}: \mathcal{N}\left(A_{+}^{*}\right)^{\perp} \rightarrow X
$$

is a bounded operator for $\gamma \leq \frac{1}{2}$.

Using (13), Theorem 2, Lemma 2, (19), and (20) we obtain

$$
\left\|v_{*}\right\| \leq \rho+C_{1} C_{*}^{2} \rho^{2} S_{n}\left(\frac{1}{2}-\gamma, 2 \gamma+\frac{1}{2}\right) \leq C_{4} \rho .
$$

Moreover, the telecopic identity (18) and (7) imply

$$
\left\|A_{+} J_{+}^{\gamma} v_{*}\right\| \leq\left\|A_{+} e_{n_{*}}\right\|+\delta \leq(1+\eta)\left(\left\|\Delta F_{n_{*}}^{\delta}\right\|+\delta\right)+\delta \leq C_{5} \delta .
$$

with $C_{5}=(1+\eta)(1+\tau)+1$. The desired bounds follow as in [3].

\section{Concluding Remarks}

In this paper we proved that the Levenberg-Marquardt regularization method converges with optimal rates under suitable assumptions. If the step sizes are chosen according to the discrepancy principle (6) proposed by Hanke [1], then it was shown in [1] that the method converges without requiring a source condition. If the source condition (cf. Assumption 1) is satisfied, then Theorem 1 shows that the rate of convergence is optimal, if the step sizes chosen by (6) do not grow faster than (9). Note that (9) is satisfied if $h_{j+1} / h_{j} \leq$ const, $j=0,1, \ldots$, so that this result appears to be relevant for practical applications. However, if (9) fails to be true, then Theorems 1 guarantees that one can switch to any step size sequence satisfying (9) and being bounded away from zero and still gets optimal convergence rates. 


\section{References}

1. Hanke, M.: A regularizing Levenberg-Marquardt scheme, with applications to inverse groundwater filtration problems. Inverse Problems 13(1), 79-95 (1997)

2. Hanke, M., Neubauer, A., Scherzer, O.: A convergence analysis of the Landweber iteration for nonlinear ill-posed problems. Numerische Mathematik 72(1), 21-37 (1995)

3. Hochbruck, M., Hönig, M., Ostermann, A.: A convergence analysis of the exponential Euler iteration for nonlinear ill-posed problems. Inverse Problems (to appear)

4. Jin, Q.: On a regularized Levenberg-Marquardt method for solving nonlinear inverse problems. Tech. rep., University of Texas at Austin (2009)

5. Jin, Q., Tautenhahn, U.: On the discrepancy principles for some Newton type methods for solving nonlinear inverse problems. Numerische Mathematik 111(4), 509-558 (2009)

6. Kaltenbacher, B., Neubauer, A., Scherzer, O.: Iterative Regularization Methods for Nonlinear Ill-Posed Problems. De Gruyter, Berlin, New York (2008)

7. Rieder, A.: On the regularization of nonlinear ill-posed problems via inexact Newton iterations. Inverse Problems 15(1), 309-327 (1999)

8. Rieder, A.: On convergence rates of inexact Newton regularizations. Numerische Mathematik 88(2), 347-365 (2001)

9. Tautenhahn, U.: On the asymptotical regularization of nonlinear ill-posed problems. Inverse Problems 10(6), 1405-1418 (1994) 